\title{
The Key Technology Research and Implementation of Alarm and Graphics Gateway in Substation Based on Embedded Platform
}

\author{
Hao Ren ${ }^{1, a}$, Xiaoqing $\mathrm{Ma}^{2, \mathrm{~b}}$, Renhui Dou ${ }^{1, \mathrm{c}}$, Zhiqiang $\mathrm{Yao}^{1, \mathrm{~d}}$ \\ ${ }^{1}$ China Electric Power Research Institute, No.8 Nari Road, Gulou District of Nanjing, 210000, China \\ ${ }^{2}$ Nari Technology Development Limited Company, No.19 Chengxin Avenue, Jiangning District of \\ Nanjing, 211106, China \\ arenhao@epri.sgcc.com.cn, ${ }^{b}$ maxiaoqing@sgepri.sgcc.com.cn, ${ }^{c}$ dourenhui@epri.sgcc.com.cn, ${ }^{\text {d } y a ~}$ \\ ozhiqiang@epri.sgcc.com.cn
}

Keywords: Alarm Direct Pass; Remote Browsing; Platform Cutting; Data Planning

\begin{abstract}
To achieve the functions of alarm direct pass and remote browsing National Electric Power Dispatching Center put forward, each substation monitoring manufacturers commonly adopted the way based on their own workstations currently, which exists some problems such as resource wasting, higher transformation cost, poor equipment reliability and so on. This paper presents a way of alarm and graphics gateway based on embedded platform, which adopts industrial-grade products, no fan, no hard drive embedded devices. From some aspects of the platform cutting, consistency of data transmission and data planning to research, some key technologies are breakthrough, and the transplantation of the embedded Linux platform and the deployment of alarm and graphics gateway function module are achieved finally. Proved by testing, the alarm and graphics gateway machine meets the functional requirements of alarm direct pass and remote browsing fully.
\end{abstract}

\section{Introduction}

In order to implement "work opinions on promoting large-scale operation system construction work comprehensively" and its technical support requirement, National Electric Power Dispatching Center points out that substation under large-scale operation must comply with the basic principle of "alarm direct pass, remote browsing, data optimization and safety certification". At the same time, the substation dispatching and control information exchange standard is published, stipulating substation should support the functions of alarm direct pass and remote browsing. In power system operation, data transmission between substation and dispatching station traditionally only has one way of telecontrol communication, relying on remote information point table through CDT or 101/104 protocol transmission. The large-scale operation system of the state grid requires the implementation of dispatching and control integration, and dispatching network and device monitoring service fusion. Therefore, a large number of substation data information is needed to send up, increasing the storage and processing burden of master station system database. To solve the problem, National Electric Power Dispatching Center put forward a new data interaction method of alarm direct pass and remote browsing, and launched the pilot, in order to solve the transmission and processing problems that substation data explosion brought about [1][2][3].

At present, to achieve the function of alarm direct pass and remote browsing, the existing solution of domestic mainstream substation secondary manufactures is adding two workstations through independent channels to achieve the function of alarm direct pass and remote browsing, causing some problems about resources waste, high construction costs and poor equipment reliability. To solve those problems, one method of alarm and graphics gateway based on embedded platform is put forward, using the embedded Linux as the system platform architecture of alarm and graphics gateway. Then, according to the functional requirement of gateway, the embedded Linux is cut and optimized, on which builds a minimized substation SCADA system, and on the basis of which deploys some functional modules associated with alarm and graphics gateway. Finally, the function of alarm direct pass and remote browsing can be realized, improving the efficiency of utilization of 
hardware resources, reducing the improvement and maintenance cost, and improving the stability and reliability of equipment.

\section{Key Technology Research}

The key technologies of alarm and graphics gateway in substation based on embedded platform mainly include the cutting and optimization technology of embedded Linux platform for realizing the transplantation in embedded equipment, the data transmission technology based on sliding window protocol for ensuring the data synchronization of transceiver in the process of communication, and the signal merging technology based on information classification and hierarchy of data planning and many associated events reasoning for optimizing and standardizing the alarm direct transferring information.

Cutting Technology Based on Embedded Linux Platform. Linux operation system has the whole system function and structure as a desktop system. In the embedded system, system should adapt to the functional requirements, providing the best possible service for users. However, because of restricted resources, both the computing power of CPU and RAM are smaller, so Linux operation system should be further cut and optimized in many aspects. Embedded Linux system generally includes Linux kernel, file system and applications, so the cutting methods of the existing Linux system mainly include three aspects accordingly, such as kernel cutting and customization, system runtime library reduction, application cutting and so on.

Because the embedded system is a specific system for specific application, the best way is to determine the kernel needs what functions support, according to the characteristics of their own hardware platform and the required software function. Through the cut, the unused codes of kernel are removed, and the kernel and function library are refined. That is the reduction method using application oriented.

Linux is similar to other single kernel UNIX operating system, composed of a lot of collaborative working function calling. So compared to the fixed programs having typical hierarchical structure, not fixed kernel structure makes the kernel structure of Linux is difficult to understand, while the function calling graph can solve this problem. The principle of function calling graph is extracting the calling relationship among each function in the process of working, presenting the function relationship structure of the whole program intuitively, in order to help us understand the kernel structure better. Here, we can abstract application, system library as well as the kernel to construct a whole function calling relationship graph, then remove the unnecessary code according to the relationship diagram, and finally the embedded Linux operation system application oriented is obtained. According to the Linux hierarchy structure, the application program is at the top of the whole structure, which can send service invocation requests to the kernel through the system library calling, as shown in Figure 1.

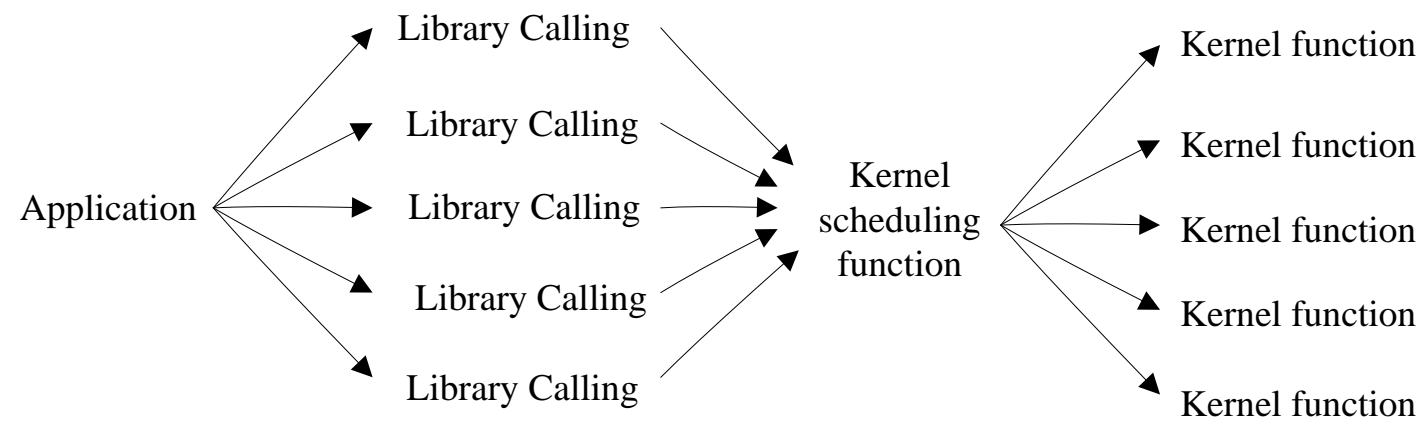

Fig.1. The Application program call kernel functions through the system library

Because the application invokes the Linux kernel function through system function library, according to the calling relations between system library and kernel function, system functions required are extracted and unnecessary functions are deleted, so the cutting and optimization of embedded Linux can be realized. 
Data Transmission Technology Based on Sliding Window Protocol. In the process of standard alarm string, graphics file and picture refreshing data packet transmitted by alarm and graphics gateway, there will be some abnormal situations such as packet corruption, packet loss, duplication of packets, premature and timeout. How to ensure the consistency of the transceiver's data is a key problem. This paper adopts the sliding window protocol to solve the reliability problem, which can realize flow control and error recovery through synchronization, retransmitting any damaged or lost data and abandoning repeated transmission data [4][5].

Sliding window protocol is divided into three protocols, including one bit sliding window, back $\mathrm{N}$ and selective retransmission. One bit sliding window method can continue to send the next frame after each frame is confirmed, reducing the channel utilization greatly. In the back $\mathrm{N}$ protocol, the receiver can no longer receive subsequent frames if the error frame is found, even though the arriving frames are correct. Obviously, this is a waste. This paper uses the sliding window method of selective retransmission protocol, which can solve the channel resource waste problem. The strategy is that when the receiver finds a frame error, even though the consequent correct frames can't be submitted to the upper layer of receiver immediately, the receiver can still receive, then store in a buffer. At the same time, the sender is required to transmit the error frame. Once the retransmitted frames are received, the rest frames stored in the buffer are submitted to the upper layer together in the right order. The method is called selective repeat, and its working process is shown in Figure 2. Obviously, selective repeat reduces waste, but requires the receiver to have very large enough buffer space.

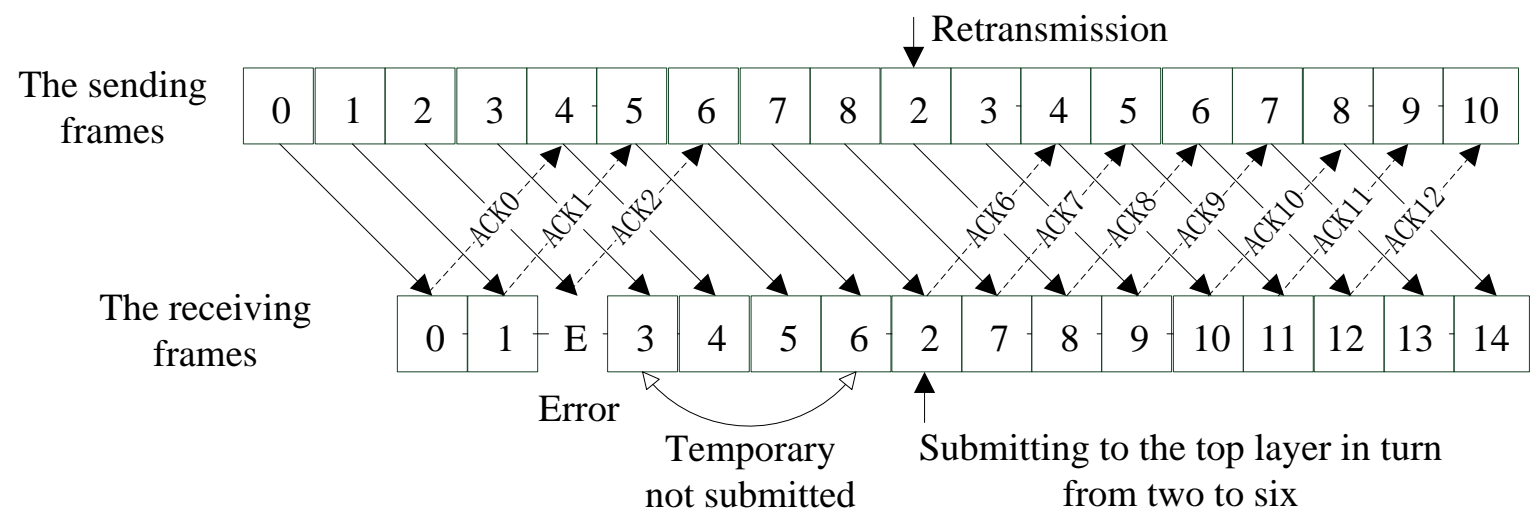

Fig.2. The working process of selective retransmission protocol

Classification and Merging Technology of Alarm Direct Transferring Information Based on Data Planning. According to the influence degree of the power grid, the alarm information is divided into five kinds, including the accident information, the abnormal information, the position change information, the off-limit information and the informing information. The accident information includes switch tripping, protection device outlet tripping and other information influencing the whole station safe movement. The abnormal information includes the alarm signal reflecting the abnormal operation situation, the signal influencing remote control operation and the important signal directly threatening to network safety and equipment operation. The position change information includes the state change signal of switch equipment. The off-limit information includes the signal the important remote measurement beyond the upper and lower limits of the alarm interval. The informing information includes the general signal reflecting the operation situation and state detection of power grid equipment [6].

In order to optimize a large amount of substation alarm information submitted by alarm direct transferring, make the operation personnel of dispatching end grasp the key point of accident, ensure the abnormal accident treated accurately and timely, improve the accuracy and fast of the exception accident handled, and guarantee the safety of power grid operation, alarm and graphics gateway needs to classify the mass of alarm information, filter a large number of suggestive informing information that the monitoring personnel do not need to concern from the source, and only preserve the accident, abnormal, position change and not reset alarm information that the persons on duty really need to be concerned. Secondly, the knowledge base of expert system for substation alarm information processing is established, single or a plurality of the related alarm events are reasoned 
and judged, and the causes and treatment measurements are given, using the forward reasoning and logic reasoning technology.

\section{Design and implementation of Scheme}

The alarm and graphics gateway includes the functions of alarm direct transmitting and remote browsing. The function of alarm direct transmitting is as following. Single event or comprehensive analysis results of substation monitoring system are put as the source of information. According to the five class classification standards of accident, abnormal, off-limit, displacement and informing, referring the standard information format of Syslog, the standard alarm strings are generated after the solutions of sorting, merging and optimizing. The standard text format alarm strings using DL476 protocol are transmitted to dispatching master station via the alarm direct transferring module. After the alarm service program of master station accesses, alarm information can be displayed classifiably on the corresponding alarm client. The function of remote browsing is as following. Using CIM/G language as the browsing graph file standard, DL476 protocol as the carrier of graph data transmission, graph file and refresh data are sent to the host station via data transferring module. After the graph program of host station accesses, substation monitoring graphics and graph refresh data can be browsed remotely. The design scheme diagram of alarm and graphics gateway is shown in Figure 3.

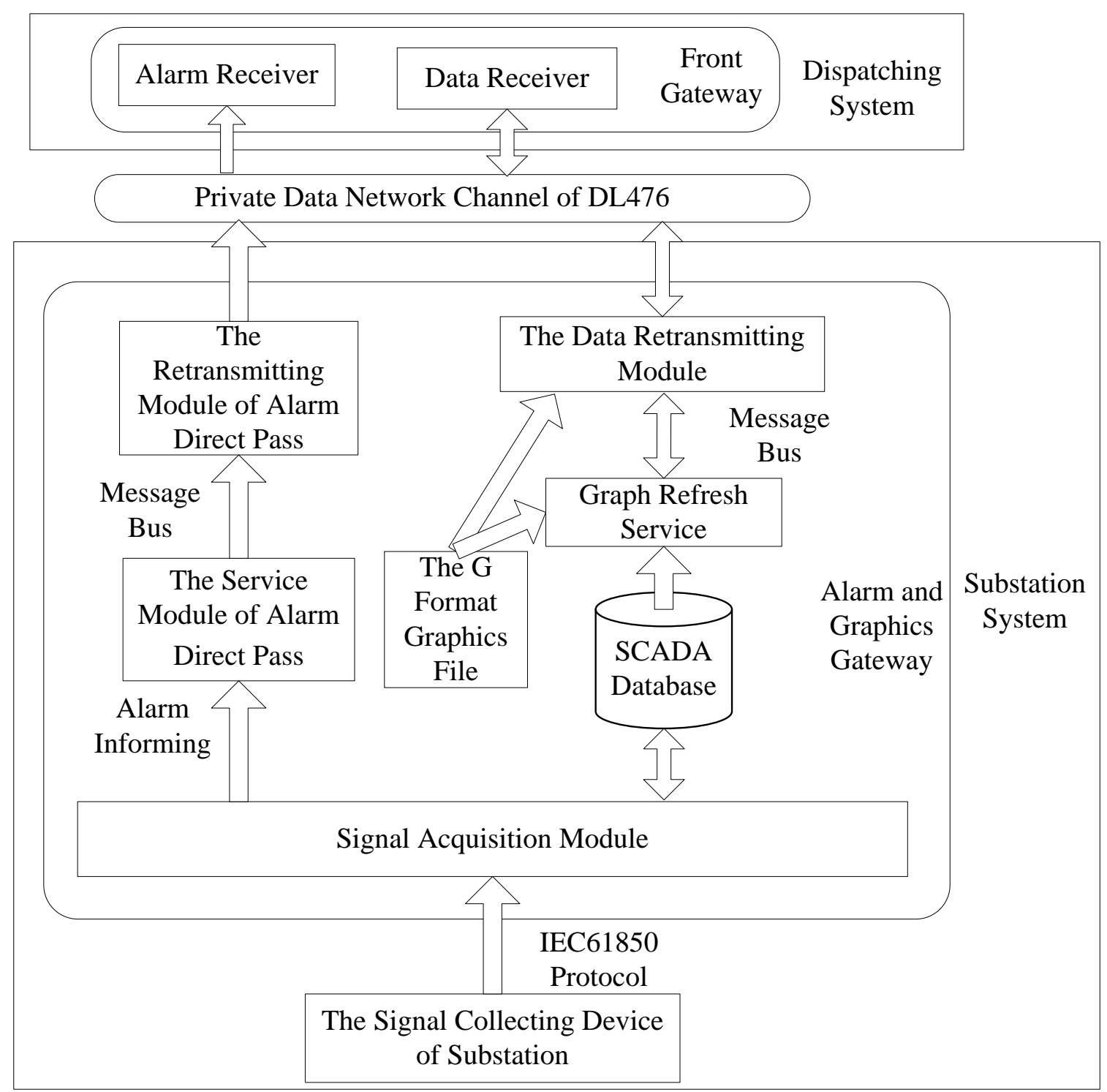

Fig.3. The design scheme diagram of alarm and graphics gateway 


\section{Test Results}

The test process of alarm direct transferring and remote browsing in embedded platform includes communication link, alarm direct transferring information sending, graph file sending, the whole data and change data of graphics sending.

In the test of alarm direct transferring function, using virtual IED simulation software to simulate protection output, protective plate, measurement and control remote / local, breaker / switch position change, abnormal alarm, communication status and remote measurement off-limit signal, the standard alarm string information is displayed in the dispatching alarm client window, and the contents of event are action / return, input / output, closing / opening, interrupt / normal, and off-limit / return. In the test of remote browsing, using virtual IED simulation software to simulate the remote signal, remote measure, and relay protection signal of CT2205 equipment in \#5 main transformer $220 \mathrm{kV}$ side monitoring picture, the static primary wiring diagram and dynamic graphics data are displayed in the dispatching D5000 graphics browsing window.

\section{Conclusion}

This paper puts forward the whole design scheme of alarm and graphics gateway based on embedded platform, on the basis of the research of key technology of alarm and graphics gateway concerning, realizing the transplantation of embedded Linux platform and the deployment of alarm and graphics gateway function module, improving the efficiency of utilization of hardware resources, reducing the maintenance cost, and improving the reliability of equipment. Proved by testing, the dispatching end can browse the alarm direct transferring information after classification, processing and optimizing, and the real time graphics and refresh data of station end at any time, realizing the direct monitoring of operation status, the trend of power flow, the position of primary device and the optical signal in substation system, and improving the efficiency of dispatching operation monitoring.

\section{Acknowledgements}

This work was financially supported by the Science and Technology Project of State Grid Corporation of China, the name of which is "The Deepening Research of Secondary Key Equipment and Integrated Business Platform in Smart Substation” (Project No. DZ71-14-035).

\section{References}

[1] WANG Lei. The application of alarm direct pass and remote browsing in Zhejiang province scheduling[J]. Transmission \& Distribution, 2012, 29(5): 52-55.

[2] HU Liqing, XIAO Yanwei, LI Ying. The technology of alarm direct pass about substation monitoring information and the application in Zhejiang power grid[J]. Zhejiang Electric Power, 2012 (12): 87-90.

[3] LIU Xifeng, WANG Yanbo, HAN Zhenfeng. The realization method of substation remote browsing technology[J]. Shandong Electric Power, 2012 (6): 41-43.

[4] IEC61850-6 Communication Networks and Systems in Substations-Part6: Configuration description language for communication in electrical substations related to IEDs[S]. Geneva: IEC, 2004-03.

[5] IEC61850-7-4 Communication Networks and Systems in Substations-Part7-4: Basic communication structure for substations and feeder equipment-Compatible logical node classes and data classes[S]. Geneva: IEC, 2003-05.

[6] LI Juntang, GU Su, CHEN Xiaohui. The research on construction scheme of remote control interactive system for 500 kV substation[J]. Electric Power Construction, 2013,34(4): 99-101. 